\title{
Surfactant-Induced Postsynthetic Modulation of Pd Nanoparticle Crystallinity
}

\author{
Yi Liu, ${ }^{+}$Chao Wang, ${ }^{*, \neq}$ Yujie Wei, ${ }^{\S}$ Leyi Zhu, ${ }^{\ddagger}$ Dongguo Li, ${ }^{+, \neq}$J. Samuel Jiang, ${ }^{\ddagger}$ Nenad M. Markovic, ${ }^{\neq}$ \\ Vojislav R. Stamenkovic, ${ }^{\neq}$and Shouheng Sun ${ }^{*,+}$ \\ ${ }^{\dagger}$ Department of Chemistry, Brown University, Providence, Rhode Island 02912, United States \\ ${ }^{\ddagger}$ Materials Science Division, Argonne National Laboratory, Argonne, Illinois 60439, United States \\ ${ }^{\S}$ State Key Laboratory of Nonlinear Mechanics, Institute of Mechanics, Chinese Academy of Sciences, Beijing, 100190, \\ People's Republic of China \\ S Supporting Information
}

ABSTRACT: Modulation of Pd nanoparticle (NP) crystallinity is achieved by switching the surfactants of different binding strengths. Pd NPs synthesized in the presence of weak binding surfactants such as oleylamine possess polyhedral shapes and a polycrystalline nature. When oleylamine is substituted by trioctylphosphine, a much stronger binding surfactant, the particles become spherical and their crystallinity decreases significantly. Moreover, the Pd NPs reconvert
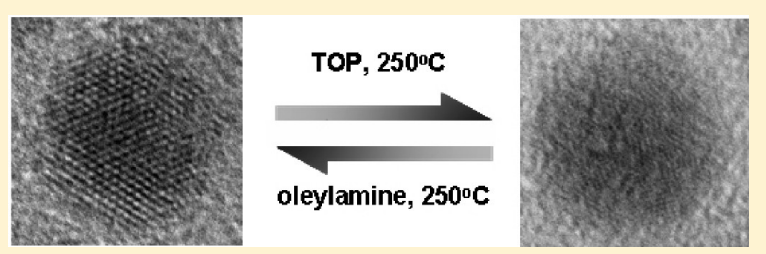
their polycrystalline structure when the surfactant is switched back to oleylamine. Through control experiments and molecular dynamics simulation, we propose that this unusual nanocrystallinity transition induced by surfactant exchange was resulted from a counterbalance between the surfactant binding energy and the nanocrystal adhesive energy. The findings represent a novel postsynthetic approach to tailoring the structure and corresponding functional performance of nanomaterials.

KEYWORDS: Palladium nanoparticle, surfactant exchange, nanoparticle structure, crystallinity modulation

\begin{abstract}
A wide range of nanomaterials are prepared by solution phase synthesis today. On the basis of the nanocrystal nucleation and growth from a homogeneous solution of the precursors, solution processing usually possesses the advantages of well control over particle size, shape, composition, and surface properties. ${ }^{1-3}$ Among various factors governing the NP growth in solution phase, ${ }^{2}$ surfactants that bind to and coat on the NP surface play a particularly important role. It is known that surfactants stabilize the NPs in solution and restrict their size in growth. Their binding selectivity on different crystal facets can also govern the anisotropic growth of NPs into various shapes such as cube, ${ }^{4,5}$ rod, ${ }^{6-9}$ and wire. ${ }^{10,11}$ Moreover, the surfactants anchored on the surface to a large extent define the surface properties of the obtained NPs and thus have to be taken into account in further functionalization of such nanomaterials. ${ }^{12,13}$ For example, ligand exchange, i.e., replacing the existing surfactant by another type, has been shown to be critical for selfassembly $^{14,15}$ and biomedical applications. ${ }^{16-18}$ For most catalytic applications, however, surfactants have to be removed so that the inorganic surface of NPs can be exposed to reactants. ${ }^{19-22}$ All of these indicate that further insights into the interplay between surfactants and NP surface are desired to acquire comprehensive understanding of NP surface chemistry and to optimize NP properties and performance for various applications.

Here we report the postsynthetic structure modulation of metallic Pd NPs induced by surfactant replacement. Pd NPs synthesized with oleylamine (OAm) as surfactant have a polyhedral
\end{abstract}

shape and a polycrystalline structure. When oleylamine is substituted by trioctylphosphine (TOP), a much stronger surfactant binding to $\mathrm{Pd}^{23}$ the NP shape is changed to near-spherical with NP crystalline size substantially reduced. The polycrystalline nature of the Pd NPs is further found to be recovered when the Pd-TOP NPs are aged in an OAm solution. After various control experiments and molecular dynamic simulations, we believe this unusual surfactant enabled modulation of NP crystallinity arises from a counterbalance between the surfactant binding energy and nanocrystal adhesive energy. Although surface-induced nanostructure transitions have been reported before, they have been limited only to metal oxides (e.g., $\left.\mathrm{ZrO}_{2}\right)^{24}$ and IIB/VIA semiconductors such as $\mathrm{ZnS}^{25-27}$ To the best of our knowledge, there has been no report on such a modulation in metallic NPs.

The synthesis of polycrystalline Pd NPs with OAm as the surfactant (denoted as $\mathrm{Pd}-\mathrm{OAm} 1$ ) was modified from a previously reported procedure. ${ }^{28} \mathrm{Pd}(\mathrm{II})$ acetylacetonate was dissolved in $\mathrm{OAm}$ at room temperature and then heated to $60{ }^{\circ} \mathrm{C}$ when a morpholine borane (MB) complex in OAm solution was injected to initiate the nucleation. Immediately after $\mathrm{MB}$ injection, the heating temperature was raised to $90^{\circ} \mathrm{C}$ for NP growth and kept at this temperature for $0.5 \mathrm{~h}$ to obtain Pd-OAm1 NPs (see the Supporting Information for details). Figure 1A shows a representative transmission electron microscopy (TEM) image of the

Received: December 30, 2010

Revised: February 13, 2011

Published: February 28, 2011 

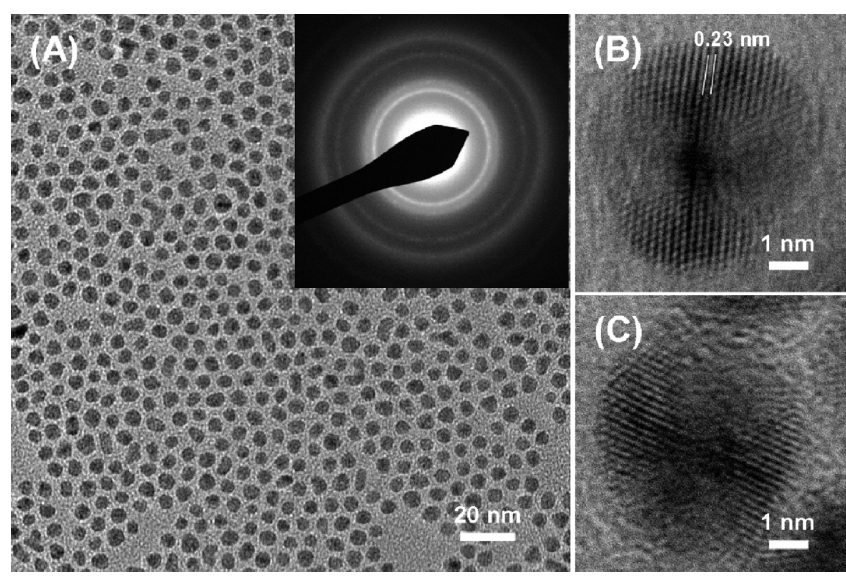

Figure 1. (A) TEM image and SAED pattern (inset). (B) and (C) HRTEM images of the as prepared $6.3 \mathrm{~nm} \mathrm{Pd-OAm1} \mathrm{NPs.}$
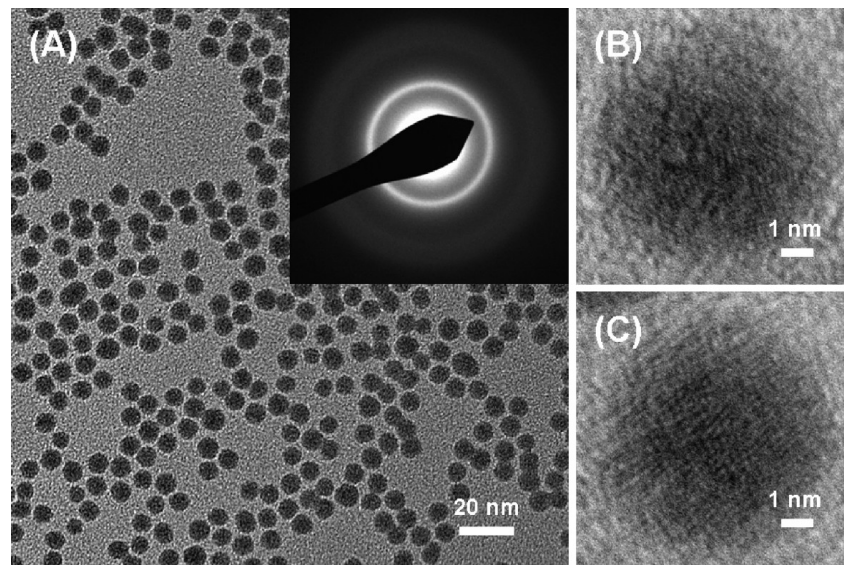

Figure 2. (A) TEM image and SAED pattern (inset). (B) and (C) HRTEM images of the as prepared $7.1 \mathrm{~nm} \mathrm{Pd-TOP} \mathrm{NPs.}$

as-synthesized monodisperse $6.3 \pm 0.6 \mathrm{~nm} \mathrm{Pd-OAm1} \mathrm{NPs}$ (Figure S1A, Supporting Information). A high-resolution TEM (HRTEM) study shows that the NPs have a polycrystalline structure (panels B and C of Figure 1). The lattice fringe was measured to be $0.23 \mathrm{~nm}$, close to the spacing of the (111) planes in face centered cubic ( $\mathrm{fcc}) \mathrm{Pd}(0.223 \mathrm{~nm})$. Selected area electron diffraction (SAED) pattern (inset in Figure 1A) shows four diffused rings, which can be assigned to the diffractions from (111), (200), (220), and (311) planes of an fcc Pd phase. The polycrystalline nature of the as-synthesized $\mathrm{Pd}-\mathrm{OAm} 1 \mathrm{NPs}$ is consistent with the results from a previous study. ${ }^{28}$

Tuning of the NP crystallinity was achieved by replacing OAm with TOP as the surfactant. After the growth of Pd NPs in oleylamine at $90^{\circ} \mathrm{C}$, TOP was added to the solution in situ. The solution temperature was then raised to $250{ }^{\circ} \mathrm{C}$ and kept at this temperature for $1 \mathrm{~h}$. The Pd NPs separated from this TOP treatment $(\mathrm{Pd}-\mathrm{TOP})$ were checked by energy-dispersive $\mathrm{X}$-ray spectroscopy (EDS) after washing with ethanol. The observation of phosphorus (P) and a $\mathrm{Pd} / \mathrm{P}$ atomic ratio of $84 / 16$ indicated the accomplishment of surfactant exchange (Figure S2A, Supporting Information). The Pd-TOP NPs have a nearly spherical shape, and their size is increased to $7.1 \pm 0.6 \mathrm{~nm}$ (Figure 2A and Figure S1B (Supporting Information)). HRTEM images (panels $\mathrm{B}$ and $\mathrm{C}$ of Figure 2) show a nanostructure with reduced crystallinity in comparison with the Pd-OAm1 NPs. The SAED

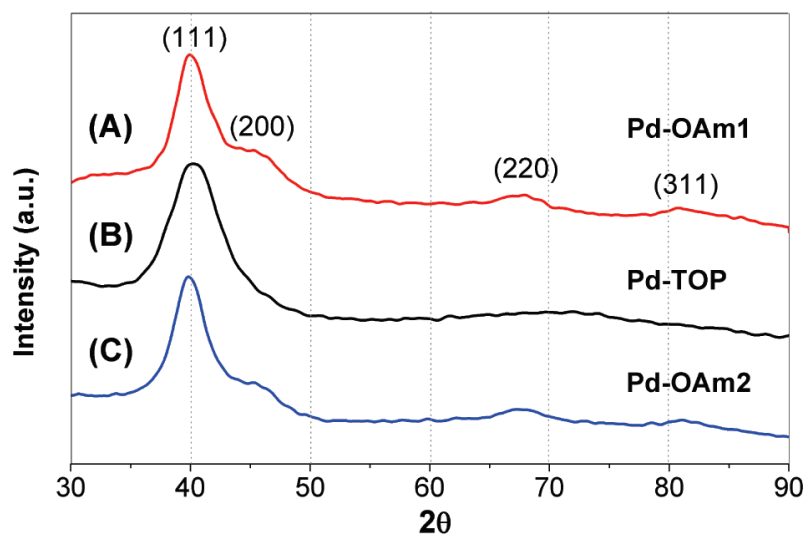

Figure 3. XRD patterns of (A) Pd-OAm1 NPs, (B) Pd-TOP NPs, and $(\mathrm{C}) \mathrm{Pd}-\mathrm{OAm} 2 \mathrm{NPs}$.
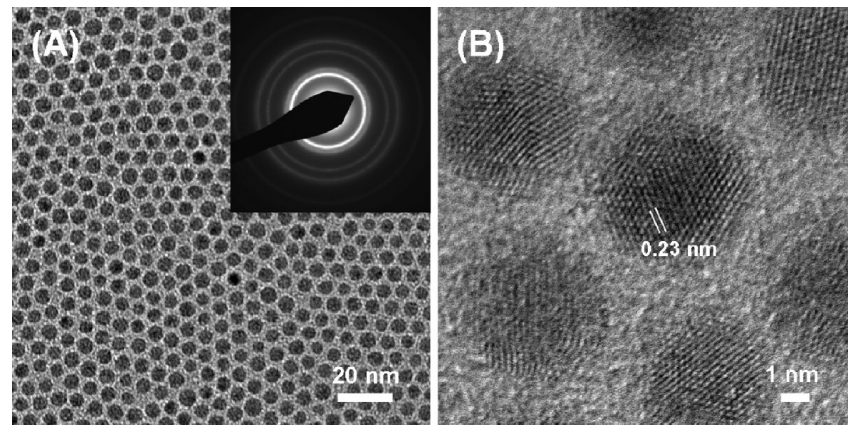

Figure 4. (A) TEM image and SAED pattern (inset). (B) HRTEM image of the as prepared $5.9 \mathrm{~nm} \mathrm{Pd}-\mathrm{OAm} 2 \mathrm{NPs}$.

pattern (inset of Figure 2A) shows only the fuzzy (111) ring. The reduced crystallinity in $\mathrm{Pd}-\mathrm{TOP}$ NPs was further characterized by X-ray diffraction (XRD) patterns (Figure 3). The $\mathrm{Pd}-\mathrm{OAm} 1$ NPs gave four characteristic diffraction peaks corresponding to (111), (200), (220), and (311) of fcc Pd, while Pd-TOP NPs show only one broader (111) peak. The increase in (111) peak width and the disappearance of the other high-angle diffraction peaks confirm that the crystalline domains in the Pd-TOP NPs became much smaller after the surfactant exchange.

Interestingly, the above structure change can be reversed when TOP is replaced again by OAm. This was done by heating the $\mathrm{Pd}-\mathrm{TOP}$ NPs in an OAm solution at $250^{\circ} \mathrm{C}$ (the obtained NPs were denoted as $\mathrm{Pd}-\mathrm{OAm} 2$ ). Though TOP binds more strongly to Pd than OAm, partial replacement of TOP by OAm on the particle surface still occurred in the OAm-rich environment at the elevated temperature, as confirmed by elemental analysis of the $\mathrm{Pd}-\mathrm{OAm} 2 \mathrm{NPs}$. EDS analyses show that the $\mathrm{Pd} / \mathrm{P}$ atomic ratio was changed from $84 / 16$ for Pd-TOP to $93 / 7$ for $\mathrm{Pd}-\mathrm{OAm} 2$ NPs (Figure S2A,B, Supporting Information). Figure 4A shows the TEM image of the $\mathrm{Pd}-\mathrm{OAm} 2 \mathrm{NPs}$. They are still in near spherical shape as in $\mathrm{Pd}-\mathrm{TOP}$ NPs but their size is slightly reduced to $5.9 \pm 0.5 \mathrm{~nm}$ (Figure S1C, Supporting Information). The HRTEM image (Figure 4B) shows the clear lattice fringes of fcc Pd (111) planes. XRD also confirms the higher crystallinity of $\mathrm{Pd}-\mathrm{OAm} 2 \mathrm{NPs}$ than $\mathrm{Pd}-\mathrm{TOP} \mathrm{NPs}$, where four diffraction peaks are observed. The crystal domain size calculated from the line broadening of (111) peak based on Scherrer's equation was $2.9 \mathrm{~nm}$ for $\mathrm{Pd}-\mathrm{OAm} 2$, close to the value $(2.7 \mathrm{~nm})$ for $\mathrm{Pd}-$ OAm1 and much larger than that $(2.0 \mathrm{~nm})$ for Pd-TOP NPs. 
The observed crystallinity change upon surfactant exchange between TOP and OAm seems to indicate that the chemical nature of the surfactant has an effect on the NP structure and crystallinity. While the detailed mechanism remains elusive, several factors were first recognized to be critical for attaining the postsynthetic modulation of NP crystallinity in solution. At first, TOP is essential for reducing the Pd NP crystallinity. Without TOP, heating the $\mathrm{Pd}-\mathrm{OAm} 1 \mathrm{NPs}$ in $\mathrm{OAm}$ at $250^{\circ} \mathrm{C}$ (for $1 \mathrm{~h}$ ) resulted in particle aggregation and increase in crystalline domain sizes (Figures S3 and S4,Supporting Information). This elevated temperature $\left(\sim 250^{\circ} \mathrm{C}\right)$ also seems to be crucial for the structure change. If the $\mathrm{Pd}-\mathrm{OAm} 1 \mathrm{NPs}$ were treated with $\mathrm{TOP}$ at $90^{\circ} \mathrm{C}$, no dramatic change was observed in particle size, shape, or crystallinity (Figures S3 and S5,Supporting Information). This could be due to the incomplete surfactant exchange at this temperature, as revealed by a higher $\mathrm{Pd} / \mathrm{P}$ atomic ratio of $96 / 4$ determined by EDS analysis (Figure S2C, Supporting Information). This indicates that the NPs treated at $90{ }^{\circ} \mathrm{C}$ have less $\mathrm{P}$ than the Pd-TOP NPs $(84 / 16)$ obtained at $250{ }^{\circ} \mathrm{C}$. Furthermore, the replacement of TOP by OAm can be identified as the key for the reversion from disordered ( $\mathrm{Pd}-\mathrm{TOP})$ to polycrystalline (Pd-OAm2) NPs. When the ligand exchange was blocked by the presence of extra TOP in the solution, the reversion process was hindered and small crystalline domains remained in the $\mathrm{Pd}$ NPs even after aging at $300{ }^{\circ} \mathrm{C}$ for $1 \mathrm{~h}$ (Figures S3 and S6, Supporting Information). It is also noticed that the Pd NPs show significant volume changes during the treatments, with $+43.1 \%$ from $\mathrm{Pd}-\mathrm{OAm} 1$ to $\mathrm{Pd}-\mathrm{TOP}$ and $-42.6 \%$ from $\mathrm{Pd}-\mathrm{TOP}$ to $\mathrm{Pd}-\mathrm{OAm} 2$. This is likely to be mainly caused by the crystallinity changes despite other possible factors like the different synthetic conditions. It is reasonable to believe that the $\mathrm{Pd}-\mathrm{TOP}$ NPs with reduced crystallinity contain a large number of defects (such as vacancy) in the particles, which might cause the substantial volumetric expansion.

Qualitative understanding of the NP crystallinity modulation was further provided by molecular dynamics simulation using the open source software Lammps distributed by Sandia National Laboratories. ${ }^{29}$ The embedded atom method potential ${ }^{30}$ for Pd was applied. All simulations were performed using NVT ensemble (or canonical ensemble, the number of atoms $(N)$, volume of the system $(V)$, and the system temperature (T) are kept constant) integration in Lammps at $600 \mathrm{~K}$. A constant time step of $1 \mathrm{fs}$ was used. We first heated the $5 \mathrm{~nm}$ particle to a high temperature (e.g., $3000 \mathrm{~K}$ ) and then cooled it quickly to $600 \mathrm{~K}$, to establish an initial amorphous state of the particle as shown in Figure 5A. By relaxing the amorphous particle in vacuum for $2 \mathrm{~ns}$, we could see that the particle crystallized (Figure 5B), implying the preferential crystallization of Pd NPs in the absence of surfactants or in the presence of a weak surfactant. However, if we applied a spherical potential wall on the particle in order to mimic the confinement introduced by the strong surfactant, ${ }^{23,25}$ we found that the potential wall exerted a surface tension on the amorphous particle, and the crystallization tendency of atoms was suppressed. The particle remained amorphous after the same relaxation ( $2 \mathrm{~ns}$ ), as shown in Figure 5C. Considering that the confinement effect by surfactant was likely to be determined by the binding energy between the surfactant and particle surface atoms, the observed reversible modulation of NP crystallinity is thus postulated to have resulted from the interplay of the surfactant binding energy and the crystal adhesive energy in the NPs. ${ }^{31}$
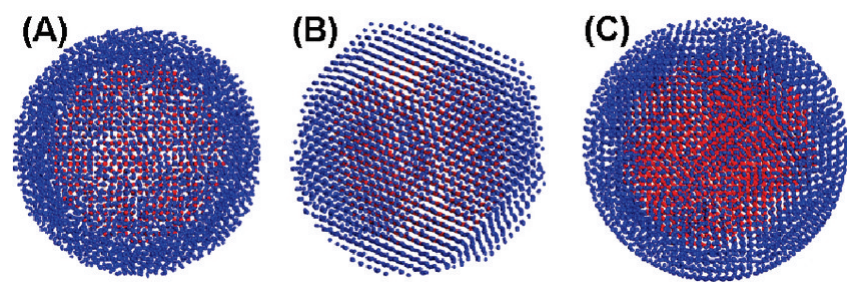

Figure 5. (A) Initial amorphous $5 \mathrm{~nm} \mathrm{Pd} \mathrm{NP} \mathrm{(blue,} \mathrm{atoms} \mathrm{at} \mathrm{the} 1 \mathrm{~nm}$ surface layer; red, inner atoms). (B) Crystallization of the amorphous Pd particle in vacuum. (C) The Pd particle remained to be amorphous if the particle was confined by surface tension.

The results from the control experiments and simulation reinforced that the binding strength of surfactants plays a key role in determining the crystallinity of Pd NPs, which could be potentially utilized to control the structure and properties of the solution processed NPs. Our preliminary studies show that the Pd NPs have distinct magnetic properties when different surfactants are present on the surface, with $\mathrm{Pd}-\mathrm{OAm} 1$ being ferromagnetic and Pd-TOP paramagnetic at $5 \mathrm{~K}$ (Figure S7, Supporting Information). ${ }^{32}$ The research thus opens a new direction for postsynthetic modulation of nanostructures and their functional performance.

\section{ASSOCIATED CONTENT}

S Supporting Information. Additional figures and experimental details. This material is available free of charge via the Internet at http://pubs.acs.org.

\section{AUTHOR INFORMATION}

\section{Corresponding Author}

*E-mail: chaowang@anl.gov, ssun@brown.edu.

\section{ACKNOWLEDGMENT}

This project was supported in part by ExxonMobil. The work conducted at Argonne National Laboratory (a U.S. Department of Energy, Office of Science Laboratory, operated by UChicago Argonne, LLC, under Contract No. DE-AC02-06CH11357) was sponsored by the U.S. Department of Energy, Office of Basic Energy Science. Y.W. acknowledges the support from the Chinese Academy of Sciences under the "Hundred Talent Program".

\section{REFERENCES}

(1) Burda, C.; Chen, X.; Narayanan, R.; El-Sayed, M. A. Chem. Rev. 2005, 105, 1025-1102.

(2) Cushing, B. L.; Kolesnichenko, V. L.; O'Connor, C. J. Chem. Rev. 2004, 104, 3893-3946.

(3) Murray, C. B.; Kagan, C. R.; Bawendi, M. G. Annu. Rev. Mater. Sci. 2000, 30, 545-610.

(4) Sun, Y. G.; Xia, Y. N. Science 2002, 298, 2176-2179.

(5) Wang, C.; Daimon, H.; Lee, Y.; Kim, J.; Sun, S. J. Am. Chem. Soc. 2007, 129, 6974-6975.

(6) Peng, X.; Manna, L.; Yang, W.; Wickham, J.; Scher, E.; Kadavanich, A.; Alivisatos, A. P. Nature 2000, 404, 59-61.

(7) Gao, J. X.; Bender, C. M.; Murphy, C. J. Langmuir 2003, 19, 9065-9070.

(8) Liu, Y.; Peng, S.; Ding, Y.; Rong, C.; Kim, J.; Liu, J. P.; Wang, Z. L.; Sun, S. Adv. Funct. Mater. 2009, 19, 3146-3150.

(9) Yin, Y.; Alivisatos, A. P. Nature 2005, 437, 664-670. 
(10) Sun, Y. G.; Gates, B.; Mayers, B.; Xia, Y. N. Nano Lett. 2002, 2, 165-168.

(11) Wang, C.; Hu, Y. J.; Lieber, C. M.; Sun, S. J. Am. Chem. Soc. 2008, 130, 8902-8903.

(12) Litran, R.; Sampedro, B.; Rojas, T. C.; Multigner, M.; SanchezLopez, J. C.; Crespo, P.; Lopez-Cartes, C.; Garcia, M. A.; Hernando, A.; Fernandez, A. Phys. Rev. B 2006, 73, No. 054404.

(13) Garitaonandia, J. S.; Insausti, M.; Goikolea, E.; Suzuki, M.; Cashion, J. D.; Kawamura, N.; Ohsawa, H.; de Muro, I. G.; Suzuki, K.; Plazaola, F.; Rojo, T. Nano Lett. 2008, 8, 661-667.

(14) Park, S. Y.; Lytton-Jean, A. K. R.; Lee, B.; Weigand, S.; Schatz, G. C.; Mirkin, C. A. Nature 2008, 451, 553-556.

(15) Grzelczak, M.; Vermant, J.; Furst, E. M.; Liz-Marzan, L. M. Acs Nano 2010, 4, 3591-3605.

(16) Choi, H. S.; Liu, W.; Misra, P.; Tanaka, E.; Zimmer, J. P.; Ipe, B. I.; Bawendi, M. G.; Frangioni, J. V. Nat. Biotechnol. 2007, 25, 1165-1170.

(17) Cheng, K.; Peng, S.; Xu, C. J.; Sun, S. J. Am. Chem. Soc. 2009, 131, 10637-10644.

(18) Yavuz, M. S.; Cheng, Y. Y.; Chen, J. Y.; Cobley, C. M.; Zhang, Q.; Rycenga, M.; Xie, J. W.; Kim, C.; Song, K. H.; Schwartz, A. G.; Wang, L. H. V.; Xia, Y. N. Nat. Mater. 2009, 8, 935-939.

(19) Wang, C.; Wang, G. F.; van der Vliet, D.; Chang, K. C.; Markovic, N. M.; Stamenkovic, V. R. Phys. Chem. Chem. Phys. 2010, 12, 6933-6939.

(20) Wang, C.; van der Vilet, D.; Chang, K. C.; You, H. D.; Strmcnik, D.; Schlueter, J. A.; Markovic, N. M.; Stamenkovic, V. R. J. Phys. Chem. C 2009, 113, 19365-19368.

(21) Chen, J. Y.; Lim, B.; Lee, E. P.; Xia, Y. N. Nano Today 2009, 4, 81-95.

(22) Peng, Z. M.; Yang, H. Nano Today 2009, 4, 143-164.

(23) Yang, Z.; Klabunde, K. J. J. Organomet. Chem. 2009, 694 1016-1021.

(24) Kirsch, B. L.; Riley, A. E.; Gross, A. F.; Tolbert, S. H. Langmuir 2004, 20, 11247-11254.

(25) Zhang, H. Z.; Gilbert, B.; Huang, F.; Banfield, J. F. Nature 2003, 424, 1025-1029.

(26) Gilbert, B.; Huang, F.; Lin, Z.; Goodell, C.; Zhang, H.; Banfield, J. F. Nano Lett. 2006, 6, 605-610.

(27) Epifani, M.; Pellicer, E.; Arbiol, J.; Sergent, N.; Pacnier, T.; Morante, J. R. Langmuir 2008, 24, 11182-11188.

(28) Mazumder, V.; Sun, S. J. Am. Chem. Soc. 2009, 131, 4588-4589.

(29) Plimpton, S. J. Comput. Phys. 1995, 117, 1-19.

(30) Foiles, S. M.; Baskes, M. I.; Daw, M. S. Phys. Rev. B 1986, 33, 7983-7991.

(31) Wang, C.; Tian, W.; Ding, Y.; Ma, Y.-q.; Wang, Z. L.; Markovic, N. M.; Stamenkovic, V. R.; Daimon, H.; Sun, S. J. Am. Chem. Soc. 2010, 132, 6524-6529.

(32) Bulk Pd itself is paramagnetic. However, in the form of NPs with organic ligands present on the surface, Pd NPs can possess ferromagnetic properties due to charge transfer from Pd to the ligands (ref 12). It is interesting that, unlike that reported before, the Pd-TOP NPs in this study did not show ferromagnetic behavior, which might be correlated to their highly disordered crystal nature and presence of amorphous layers on the surface (See, for example: Shinohara, T.; et al. Phys. Rev. Lett. 2003, 91, No. 197201). It might also be possible that the Pd-TOP NPs are superparamagnetic, but the anisotropy is so low that thermal fluctuations overcome the anisotropy to make the magnetization appear paramagnetic. 